\title{
Recherche \\ d'un programme optimisé de lutte contre la pollution dans le bassin de la Vire
}

\author{
par Z. Wilczynski \\ Chargé d'études \\ Agence Financière de Bassin \\ Seine-Normandie
}

\author{
ef $R$. Bujon \\ Ingénieur principal, \\ SÉTUDE, 27, Boulevard des ltaliens \\ 75002 Paris
}

L'ensemble formé par le Cotentin, le Bessin et le Bocage Normand enveloppe grossièrement les limites du ternitoire qui nous préoccupe.

Cette région s'inscrit administrativement dans les départements du Calvados et de la Manche. Chacune de ces petites régions possède une physionomie qui lui est propre mais dont le point commun est d'appartenir au pays de I'herbe. Le Cotentin et le Bessin sont des pôles herbagers; ils se spécialisent dans les produits laitiers, traités par des coopératives ou des Sociétés. Le Cotentin ajoute des élevages de sélection et des Haras.

Le Bocage Normand se prête 'à l'élévage et à la culture du pommier à cidre; lait et alcool ont été longtemps la principale ressource de petites exploitations dispersées en fermes isolées.

Les régions côtières avec leurs sables alluvionnaires hébergent d'importantes conchylicultures (moules, coques, coquilles Saint-Jacques, etc.).

Une telle situation, liée à l'expansion économique permet de mieux comprendre le grave problème qui est apparu au niveau de l'environnement.

L'étude qui va vous être exposée par M. Bujon, concerne uniquement le bassin hydrographique de la rivière Vire. Son bassin versant occupe une superficie de $1245 \mathrm{~km}^{2}$ et son cours se développe sur $116 \mathrm{~km}$ de long. Les principales sources de pollution sont observées d'amont en aval après Vire, Condé-sur-Vire et Saint-Lô. Elles sont la conséquence essentielle d'une urbanisation en plein développement et d'une industrie laitière très importante. On peut noter qu'elles menacent sérieusement la faune aquatique ainsi que les gisements coquilliers de la baie des Veys qui représentent pour cette région, une ressource économique non négligeable.

L'état de pollution aigu de la Baie des Veys, exutoire naturel des bassins de la Vire, la Douve, la Taute, et l'Aure, constaté au début de l'année 1969, avait conduit le Conseil des Ministres réuni le 10 juin 1970, à inscrire

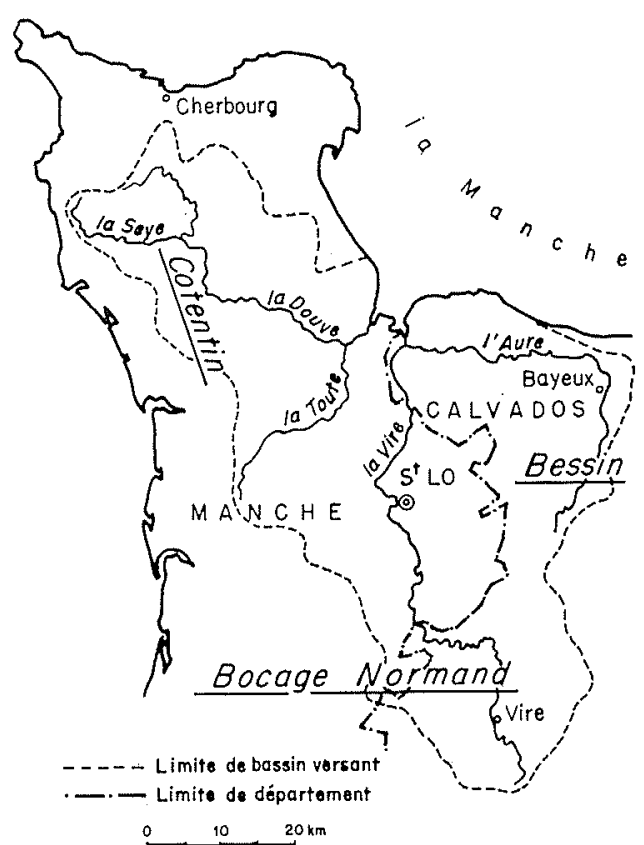

1/

la reconquête de la rivière Vire parmi les cent mesures relatives à l'environnement et à en faire une opération pilote de lutte contre la pollution.

Depuis le début de l'opération, un certain nombre de réalisations ont été effectuées: études, renforcement des ressources en eau, équipement en stations d'épuration.

La situation constatée en 1969 a beaucoup évolué. En effet, on peut considérer qu'à ce jour $60 \%$ de la pollution est traitée ou en voie de l'être (concours lancés). Le tableau suivant indique l'état d'avancement de l'équipement en stations d'épuration. 


\begin{tabular}{|c|c|c|c|}
\hline & \multicolumn{3}{|c|}{$\begin{array}{l}\text { POLLUTION ÉLIMINÉE } \\
\text { (en tonnes/jour) }\end{array}$} \\
\hline & $\begin{array}{l}\text { Domes- } \\
\text { tique }\end{array}$ & $\begin{array}{l}\text { Indus- } \\
\text { trielle }\end{array}$ & Totale \\
\hline $\begin{array}{c}\text { Stations d'épuration réalisées } \\
\text { en } 1971 \ldots \ldots \ldots \ldots \ldots \ldots\end{array}$ & 1,70 & 9,50 & 11,20 \\
\hline $\begin{array}{l}\text { Stations d'épuration réalisées } \\
\text { en } 1972 \ldots \ldots \ldots \ldots \ldots\end{array}$ & 1,60 & 6,99 & 8,59 \\
\hline $\begin{array}{l}\text { Stations d'épuration dont le } \\
\text { concours est lancé } \ldots . . . .\end{array}$ & 3,93 & 14,07 & 18,00 \\
\hline $\begin{array}{c}\text { Total } \ldots \ldots \ldots \ldots \text {. } \\
(1971+1972+\text { Projet })\end{array}$ & 7,23 & 30,56 & 37,79 \\
\hline Ouvrages à réaliser $\ldots . \ldots$. & 12,77 & 9,44 & 22,21 \\
\hline Total de lopération ...... & 20,00 & 40,00 & 60,00 \\
\hline
\end{tabular}

Pour ce qui nous préoccupe ici: les différentes études engagées ont pour but d'estimer la pollution des bassins de l'Opération Pilote et d'en suivre l'évolution au fur et à mesure de la mise en cuvre des ouvrages d'épuration.

L'Agence Seine-Normandie a confié, en particulier, à la SETUDE une étude devant permettre de déduire, compte tenu du dispositif d'assainissement existant dans le bassin de la Vire, les améliorations à apporter de manière à ce que l'eau de cette rivière réponde à l'étiage aux objectifs de qualité désirés.

Cette étude s'inscrit dans la politique d'objectifs de qualité mise en cuvre par l'Etat (circulaire interministérielle du 29 juillet 1971) qui consiste à fixer des critères de qualité selon chaque type de cours d'eau et, en conséquence, de déterminer les flux de pollution maxima à tolérer pour les rejets publics et privés.

Je passe maintenant la parole à $M$. Bujon qui va vous exposer les travaux réalisés par la SETUDE dans le cadre de l'opération pilote de la Vire.
Ainsi que l'a indiqué M. Wilczynski, l'objectif essentiel de l'étude qui nous avait été confiée consistait à définir les mesures d'assainissement à adopter ou dans certains cas les compléments d'épuration à prévoir, de telle sorte que la Vire présente en tout point de son cours soit les caractéristiques dites de la classe $1 \mathrm{~B}$ soit celles de la classe $1 \mathrm{~A}$.

Par classe $1 \mathrm{~B}$, on entendait une teneur en $\mathrm{DBO}_{5}$ inférieure à $5 \mathrm{mg} / 1$ et une teneur en oxygène dissous supérieure à $5 \mathrm{mg} / 1$ et par classe $1 \mathrm{~A}$, une $\mathrm{DBO}_{5}$ inférieure à $3 \mathrm{mg} / 1$ et une teneur en oxygène dissous supérieure à $7 \mathrm{mg} / 1$.

Le problème posé consistait donc à déterminer par le calcul les valeurs de $\mathrm{DBO}_{\tilde{5}}$ et de teneur en oxygène dissous du cours d'eau pour différentes hypothèses de degré d'épuration des stations urbaines et industrielles dont la pollution résiduelle parvient dans la Vire.

$$
* *
$$

Pour mener à bien ces calculs de $\mathrm{DBO}_{5}$ et d'oxygène dissous qui sont longs, fastidieux et, par conséquent, sources éventuelles d'erreurs, il a été utilisé un programme de calcul sur ordinateur établi par la SETUDE basé d'une part sur la loi de biodégradation classique suivante:

$$
d l / d t=-K_{1} L
$$

dans laquelle $L$ est la charge polluante exprimée en $\mathrm{DBO} \infty$ (la $\mathrm{DBO} \infty$ est proportionnelle à la $\mathrm{DBO}_{\bar{n}}$ ).

$K_{1}$ est un coefficient dit de biodégradation; $t$ est le temps

et, d'autre part, sur la loi de Streeter et Phelps selon laquelle la différentielle du déficit en oxygène par rapport au temps, est la différence de deux termes, l'un la consommation d'oxygène par les bactéries qui biodégradent la charge polluante, l'autre la réoxygénation par l'oxygène atmosphérique :

$$
d D / d t=K_{1} L-K_{2} D
$$

où $D$ est de déficit en oxygène,

$K_{3}$ est un coefficient dit de réoxygénation.

Le programme de calcul en question est ainsi conçu pour calculer la $\mathrm{DBO}_{5}$ et le déficit en oxygène dissous au fil de l'eau à partir de conditions initiales données $\left(\mathrm{DBO}_{\overline{5}}\right.$ et déficit initiaux, débit du cours d'eau, etc.), mais il permet également de faire intervenir l'influence de certains «incidents » rencontrés, par exemple:

- les affluents du cours d'eau ou les rejets qui y sont déversés;

- les barrages qui sont sources d'une réoxygénation supplémentaire;

- les stations de pompage qui diminuent le débit;

- les stations éventuelles de réoxygénation artificielles.

En outre, il est possible de faire varier à volonté la températures des eaux.

Mais, pour utiliser ce programme de calcul, il faut connaître la valeur numérique de nombreux paramètres ce qui nécessite en particulier l'exécution de mesures sur le terrain.

$$
\text { *is }
$$

Il a donc été procédé à une campagne de mesures sur la Vire. Deux sortes de renseignements étaient nécessaires: des renseignements de nature hydraulique et des renseignements de nature physico-chimiques.

Les renseignements hydrauliques correspondaient au débit et à la vitesse d'écoulement de la Vire en différents points de son cours et au débit de ses principaux affluents à proximité immédiate de la confluence.

En outre, le cours d'eau étant jalonné d'un grand nombre de barrages, la mission sur le terrain était destinée à relever leurs principales caractéristiques qui ont notamment permis d'évaluer ensuite les durées de séjour de l'eau en leur sein. De la connaissance des vitesses d'écoulement dans les tronçons à écoulement libre et des durées de séjour dans les barrages ont pu être tirés les temps 
d'écoulement moyens de l'eau de la rivière correspondant aux débits mesurés.

Les renseignements de nature physico-chimiques ont été obtenus en prélevant des échantillons et en procédant à leur analyse. Les points de prélèvement avaient été soigneusement choisis de manière à ce qu'il soit possible de déterminer sans trop de difficulté à partir des résultats de mesure, les coefficients de biodégradation $K_{1}$ et de réoxygénation $K_{2}$. La plupart étaient situés sur la Vire à l'amont et à l'aval immédiats des principales sources de pollution à savoir Vire, Condé-sur-Vire, Saint-Lô, Pont Hébert-La Meauffe, et sur ses principaux affluents juste avant confluence.

Les déterminations analytiques ont été effectuées sur des échantillons prélevés périodiquement et sur des échantillons moyens représentatifs d'une journée d'écoulement, constitué à partir de ceux-ci. Le programme analytique a comporté les déterminations des analyses de pollution type mais un grand soin a été porté à la mesure de la température, de la teneur en oxygène dissous, de la $\mathrm{DBO}_{5}$ et de la D.C.O. qui ont toutes été effectuées in situ sur des échantillons frais.

Les résultats analytiques ont été utilisés pour déterminer la valeur numérique de $K_{1}$ et de $K_{2}$. Compte tenu des variations importantes du régime d'écoulement de la Vire de l'amont à l'aval, cette détermination a été faite dans quatre tronçons distincts couvrant la totalité du cours de la rivière, et ceci grâce au programme de calcul lui-même utilisé, si l'on peut dire, «à l'envers".

$$
\vdots
$$

Outre, les débits, les temps de transit de l'eau, la pollution initiale de la Vire ou celle de ses affluents à la confluence les coefficients $K_{1}$ et $K_{2}$, il convenait également de connaître l'importance des charges polluantes générées par les Communes et les industries, de définir le débit de la rivière à prendre en compte dans les calculs prévisionnels et de déterminer les durées de transit de l'eau pour ce débit.

Les charges polluantes générées ont été déterminées à l'aide de renseignements en possession de l'Agence de Bassin, renseignements qui permettaient par ailleurs de calculer le rendement des stations d'épuration existantes.

En ce qui concerne le débit à prendre en compte, il convenait naturellement de retenir un débit de basses eaux. On ne disposait pour cela que des résultats d'une seule station de jaugeage, celle de Vire, située tout à l'amont de la rivière étudiée. L'examen des résultats des dix dernières années de mesure a permis de relever de très faibles valeurs de débit d'étiage à Vire, mais étant donné que la pollution ne provoque de nuisances graves que si le débit ne se maintient à faible valeur que pendant un temps suffisamment long, on a été amené à considérer non seulement les faibles valeurs de débit mais aussi la durée pendant laquelle ces débits se maintiennent.

Pour cette raison, il a été décidé de choisir comme débit d'étude à Vire, débit d'étude que nous appellerons dans la suite débit d'étiage par commodité, la moyenne arithmétique la plus faible de dix mesures de débit quotidiennes consécutives, soit $340 \mathrm{l} / \mathrm{s}$.

Mais, il ne suffisait pas de connaître le débit d'étiage à Vire, il fallait également déterminer le débit en tout autre point du cours d'eau dans les mêmes conditions hydrologiques. Pour cela, il a été procédé à une reconstitution du débit d'étiage de la rivière en calculant les diverses valours de débit à l'aval de la confluence de chacun des affluents: le calcul a été fait en utilisant des coefficients de débit spécifiques déduits de quelques mesures effectuées antérieurement en divers points du cours d'eau, débits spécifiques que l'on a multipliés par la superficie $\mathrm{du}$ bassin versant de chacun des affluents pour obtenir dans chaque cas l'augmentation du débit de la Vire.

En dernier lieu, ont été déterminées les durées de transit de l'eau pour les débits d'étiage, en faisant le quotient des distances parcourues par les vitesses d'écoulement, ces dernières ayant été calculées dans le cas des barrages, par le rapport volume/débit et dans les tronçons d'écoulement libre par la relation vitesse/débit que l'on peut tirer de la formule de Strickler.

$$
\text { * }
$$

Une fois connues, les valeurs numériques des paramètres que nécessite l'utilisation du programme de calcul, il a été procédé à la recherche des solutions d'épuration à adopter.

Dans un premier temps, on a demandé à l'ordinateur de calculer l'évolution de la $\mathrm{DBO}_{\overline{5}}$ et du déficit en oxygène dissous à l'étiage en supposant que tous les effluents étaient soumis à un traitement d'épuration d'une efficacité de $75 \%, 80 \%, 85 \%, 90 \%$ et $95 \%$.

Les résultats obtenus ont principalement montré que même pour le taux d'épuration le plus élevé, la $\mathrm{DBO}_{5}$ de la rivière à l'aval du rejet de la ville de Vire était encore élevée en raison du débit particulièrement faible de la rivière dans sa partie amont et que la pollution d'une distillerie située à Tessy-sur-Vire provoquait également une pointe de pollution importante ne permettant pas de respecter les objectifs de qualité requis.

C'est pourquoi, dans un second temps a été étudiée l'amélioration de qualité qu'apporterait la mise en œuvre d'un traitement d'épuration complémentaire plus ou moins poussé à Vire (ce traitement pouvant être relativement simple et consister en un lagunage de finition), les autres stations procurant les mêmes taux d'abattement que ceux précédemment indiqués et la distillerie stockant ses effluents épurés dans des réservoirs existants pour ne les rejeter qu'en période de hautes eaux (le rejet de l'effluent brut ne pouvait être retenu car il aurait provoqué une augmentation de $\mathrm{DBO}_{5}$ de 2 à $3 \mathrm{mg} / \mathrm{l}$.)

Dans ces conditions, il a été trouvé qu'en ce qui concerne la teneur en oxygène dissous l'objectif de qualité $1 \mathrm{~A}$ $(7 \mathrm{mg} / \mathrm{l})$ est atteint dans tous les cas.

En ce qui concerne la $\mathrm{DBO}_{5}$, il est apparu difficile d'obtenir en tout point de la rivière les teneurs désirées et principalement celle de l'objectif de qualité $1 \mathrm{~A}$, les zones critiques se trouvanr situées à l'aval du rejet de la ville de Vire et dans la partie inférieure du cours d'eau à cause de la pollution résiduelle de rejets successifs, relativement proches les uns des autres et assez régulièrement répartis.

Ainsi pour atteindre l'objectif de qualité $1 \mathrm{~B}\left(\mathrm{DBO}_{5}\right.$ $<5 \mathrm{mg} / \mathrm{l})$, il faudrait mettre en cuvre un dispositif d'épuration qui correspond déjà au traitement tertiaire (pourcentage d'abattement des charges polluantes minimum: 90 à $95 \%$, le dispositif devant naturellement être encore plus performant si l'on vise l'objectif de qualité $1 \mathrm{~A}\left(\mathrm{DBO}_{5}<3 \mathrm{mg} / 1\right)$.

Il ne serait cependant pas raisonnable du point de vue 
pratique de considérer que l'eau de la rivière ne correspond à tel ou tel objectif de qualité parce que sur un pourcentage relativement faible de son cours, sa qualité est quelque per moins bonne que celle recherchée.

L'exploitation de cette idée a conduit à constater que $84 \%$ de la longueur de la rivière répondraient à l'objectif de qualité $1 \mathrm{~B}$, si l'effluent de la station de Vire était épuré jusqu'à une $\mathrm{DBO}_{5}$ de $15 \mathrm{mg} / 1$, les autres effluents étant épurés à $85 \%$ et le rejet de la distillerie se faisant en période de hautes eaux. Le pourcentage de longueur tomberait à $82 \%$ si l'effluent de Vire n'était porté qu'à $25 \mathrm{mg} / 1$.

Elle a également conduit à remarquer que l'objectif de qualité $1 \mathrm{~A}$ serait atteint sur $76 \%$ de la longueur du cours d'eau si la $\mathrm{DBO}_{5}$ de l'effluent de Vire était réduite à $15 \mathrm{mg} / 1$, les autres stations d'épuration procurant un degré d'épuration de $95 \%$.

En définitive, l'étude a montré qu'en ce qui concerne la $\mathrm{DBO}_{5}$ il n'apparaît pas trop difficile de mettre en place un dispositif d'épuration susceptible de limiter, à l'étiage, la pollution de la plus grande partie de la rivière au niveau de l'objectif $1 \mathrm{~B}$. Par contre, une limitation de la $\mathrm{DBO}_{5}$ au niveau de l'objectif $1 \mathrm{~A}$ sur une longueur conséquente de la rivière nécessiterait la mise en œuvre d'un système d'assainissement très élaborée d'une efficacité supérieure à $95 \%$ et faisant appel, par conséquent, au traitement tertiaire dans tous les cas.

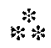

En conclusion, on voit que l'utilisation d'un tel programme facilite considérablement ce genre d'étude qui implique l'examen d'un nombre élevé de variantes, chacune d'elles nécessitant l'exécution de calculs importants qui seraient irréalisables par tout autre moyen.
On peut, certes, faire remarquer que le modèle mathématique pris en considération dans le programme ne traduit que partiellement les phénomènes naturels qui interviennent dans un cours d'eau.

On peut répondre à cette objection que la loi Streeter et Phelps prend en considération le phénomène essentiel à savoir la biodégradation par les microorganismes aérobies et la réaération par l'oxygène atmosphérique.

Certains auteurs ont proposé de perfectionner ce modèle en lui adjoignant des termes relatifs à la réoxygénation par photosynthèse, à la consommation d'oxygène par les boues de fond et même des termes relatifs à la consommation d'oxygène par les composés organiques azotés.

Le programme de calcul établi par la SETUDE offre ces possibilités de variantes. Mais tous ces perfectionne. ments ne portent que sur des phénomènes du second ordre: la réoxygénation par photosynthèse n'est intense qu'à certaines périodes de l'année qui ne correspondent pas obligatoirement à l'étiage, les boues de fond ne sont pas déposées uniformément sur le lit du cours d'eau et elles se déplacent fréquemment, la consommation d'oxygène relative à la biodégradation des composés organiques azotés n'intervient que lorsque les matières hydrocarbonées ont disparu en grande partie.

En outre, la prise en considération de ces phénomènes annexes accroît la liste déjà longue, comme on l'a vu ciavant, des informations de base dont il faut disposer pour mettre le programme en application. Compte tenu de l'imprécision inévitable qui affecterait les informations supplémentaires requises, il n'est pas certain qu'on améliorarait, tous comptes faits, la précision des prévisions en utilisant le programme de caloul dans isa version sophistiquée.

\section{Discussion}

Président : M. C. Lefrou

Merci, Monsieur, pour votre intéressant exposé, dit M. le Président. Vous avez répondu par avance, à la fin de celui-ci, à quelques questions qui viennent à l'esprit sur la validité de votre modèle et aux objections que l'on fait couramment à l'équation que vous avez utilisée.

Je voudrais avant de donner la parole à la salle vous demander sur combien de séries de valeurs a été calculé votre modèle? Ces valeurs correspondaient-e'les à des conditions naturelles différentes ou, au contraire, à des mesures eflectuées au cours d'une même période? D'autre part, avez-vous pu vérifier que, dans des conditions différentes, votre modèle donnait une bonne image de la réalité ?

M. Bujon répond :

C'est, en effet, un point important que de caler le modèle, surtout lorsque le nombre de termes est important. Notre programme a été établi il y a maintenant deux ans environ et a été utilisé à d'autres études - un peu du même genre que celle que j'ai décrite - dans le cadre de la pollution de la Vire.

Pour chaque application, nous avons bien évidemment vérifié, en fournissant à l'ordinateur tous les paramètres numériques nécessaires au calcul de la teneur en oxygène dissous et la teneur en $\mathrm{DBO}_{5}$ que nous retrouvions bien sur le listing foumi par l'ordinateur, les mesures correspondantes faites sur le terrain.
Pour retrouver les mesures faites in situ, interroge M. le Président, avez-vous introduit dans l'ordinateur uniquement des données différentes de celles qui avaient servi précédemment au calage du modèle?

M. BuJon répond par l'affirmative. Nous n'avons introduit dans le programme de calcul, dit-il, que la loi essentielle de biodégradation, les lois de réoxygénation par l'interface et dans les barrages, la loi des mélanges, à l'exclusion des données numériques qui nécessitent une détermination expérimenta'e préalable pour chaque rivière particulière à partir de mesures in situ et l'emploi à l'envers du programme.

En définitive, observe M. THIRrIot, vous considérez votre modèle mathématique plutôt comme un modèle analogique qu'un modèle représentant la réalité physique. Je pense que cela est un point intéressant car cela permet de faire comme vous le signaliez, une impasse sur certains paramètres en incorporant leur effet dans celui d'autres paramètres micux adaptés.

J'aimerais bien avoir une confirmation de votre point de vue sur cette distinction entre modèle mathématique - qui serait représentatif parce que se fondant sur des lois physiques - et modèle analogique.

Par ailleurs, vous avez dit au cours de l'exposé que vous faisiez 
fonctionner le programme à l'envers pour résoudre le «problème inverse ». Or, à Toulouse, une équipe s'occupe de modèles de pollution et travaille à peu près dans le même état d'esprit; elle a essayé notamment d'ajouter quelques autres paramètres sans trop se faire d'illusion sur la meilleure représentation de la réalité qu'elle obtiendrait ainsi; cette équipe a eu beaucoup de difficultés pour résoudre ce «problème inverse» et je voudrais que vous nous disicz si, réellement, vous utilisez ce programme à l'envers, car je ne vois pas très bien bien comment on procède.

On ne peut évidemment pas considérer, répond $M$. Bujon, qu'un modèle mathématique, utilisant les deux seules relations fondamentales que nous avons prises en compte, représente exactement la réalité. Il ne s'agit donc pas d'un modèle mathématique, mais d'un modèle analogique que nous considérons comme un outil de calcul; un certain nombre de paramètres et de phénomènes ne sont pas pris en comptes, mais nous admettons qu'en déterminant expérimentalement la valeur de certains coefticients, tels que $K_{1}$ et $\mathbf{K}_{2}$, hous intégrons un certain nombre de ces phénomènes (par exemple, celui de la consommation d'oxygène par les matières azotées).

J'en viens maintenant à la résolution du «problème inverse».

En fait, il s'agissait pour nous de déterminer $K_{1}$ et $K_{2}$, à partir de mesures qui avaient été faites en calculant leurs valeurs entre deux coup'es de points de mesures. Pour cela nous avons utilisé le programe à l'envers en ce sens que nous avons donné à l'ordinateur toutes les données nécessaires et lui avons demandé de nous fournir la valeur de $\mathrm{K}_{1}$ et celle de $\mathrm{K}_{2}$ qui permetraient de retrouver les teneurs en $\mathrm{DBO}_{5}$ et en oxygène dissous, observées aux deux stations de mesures considérées.

Il est bien évident que, du fait de cette façon d'opérer, le modèle n'est calé que dans le cas particulier du cours d'eau ou du secteur de cours d'eau sur lequel on a procédé aux dites mesures.

M. SERPaUd apporte d'intéressantes informations sur les études poursuivies par Sogreat et Alsthom Technique des Fluides, Grenoble, pour le compte de la D.G.R.S.T. - à peu près à la même époque que celles décrites par le conférencier - mais dans une perspective g'obale et avec des buts assez difiérents.

On recherchait, en prenant en compte un certain nombre de paramètres, les moyens de déceler les états de pollutions extrêmes et de prévenir les accidents qu'ils peuvent provoquer (par exemple, mort de milliers de poissons par temps chaud en étiage).

A cet effet, trois campagnes de mesures, réparties sur une durée d'un an, ont été effectuées sur la Vienne, à l'aval de Saillat; elles comportaient des relevés de débit, de températures et une série d'observations biologiques assurées par l'I.R.C.H.A. (mesures de la $\mathrm{DBO}_{5}$ et de la $\mathrm{DBO}_{20}$, notamment).

La prise en compte de nombreux paramètres ne complique pas énormément le programme de calcul mais rend plus longues et plus onéreuses les campagnes de mesures sur le terrain. Certains facteurs considérés comme nég'igeables peuvent avoir une importance insoupçonnée; c'est ainsi que nous avons été frappé par la différence entre les valeurs de la $\mathrm{DBO}_{\mathbf{5}}$ et de la $\mathrm{DBO}_{20}$.

Ce dernier point me paraît important, souligne M. le Président. Il n'est pas évident, en effet, que lorsqu'on aura réalisé une épuration à 90 ou $95 \%$, la substance polluante qui restera encore présente dans les eaux de la rivière, aura les mêmes caractéristiques que celle observée avant l'épuration; en particulier, le terme $\mathrm{K}$ restera-il le même? L'expérience de nos collègues anglais sur la Tamise a montré que l'extrapolation, dans ces conditions, des paramètres conduit parfois à quéques déboires.

Répondant à M. Serpaud, M. Bujon s'exprime en ces termes : il semblerait que dans la mise au point de votre programme, vous vous soyez engagés dans la voie de la complexité. Il s'agit de savoir si la complexité, en dehors de quelques cas de recherche pure, conduit à des résultats pratiques meilleurs.

Mais les utilisateurs de ce genre de programme complexe rencontrent beaucoup de difficulté pour déterminer la valeur numérique de paramètres en nombre multiple. Il est très difficile de réunir dans une campagne de mesures faite dans un laps de temps relativement court et avec des moyens financiers limités, toutes les informations nécessaires avec une précision suffisante. L'utilisation de paramètres complémentaires avec des va'eurs fausses ne peut que détériorer la qualité du résultat.

J'ai dit, tout à l'heure, qu'un certain nombre de facteurs pollvaient être considérés comme d'importance secondaire; je n'ai pas voulu dire par là qu'ils étaient négligeables; mais peut-être l'augmentation des dépenses et des délais nécessaires à l'acquisition des va'eurs numériques risque de compenser le supplément de précision que l'on pourrait attendre d'un programme comportant un plus grand nombre de paramètres.

M. le Président se voit dans l'obligation d'interrompre la discussion en raison des exigences de l'horaire. Il donne la parole à M. Gleizes pour l'exposé de la communication qu'il a rédigé en co!laboration avec MM. J. Delmas et A. GRAUBY. 